\title{
Landscape and the environment
}

\author{
Krystyna Wilkoszewska \\ (Institute of Philosophy, Jagiellonian University)
}

\begin{abstract}
The paper analyzes the concept of landscape and its various embodiments in art and nature. On the one hand, one can claim that our understanding of the landscape is constituted by conceptual oppositions like human/non-human, artifactual/natural, culture/nature; on the other, one may notice that landscapes occur in the space "between" these oppositions. Furthering this observation, I lodge an objection to the approach of certain exponents of environmental aesthetics who opt for replacing the notion of landscape by that of environment because I would argue that the former is still informative.
\end{abstract}

Keywords:

Aesthetics, art, environment, landscape

\section{From nature as nature to nature as culture}

Although we distinguish various kinds of landscape like, for instance, industrial landscape or urban landscape, in general human understanding, landscape is, first of all, a natural landscape, a fragment of nature understood as free from any presence, interference, or control on the part of man. Thus, we have to do with the first conceptual opposition of what is human and what is non-human. The sphere of what is human comprises man himself and all his products, while within the non-human sphere we find inanimate matter, plants, and animals. The objects produced by representatives of nature, like birds' nests, animals' burrows, or beavers' dams also belong to the non-human sphere.

Let us start, then, with the concept of "nature as nature." In the recent years it has frequently been written that nature as nature does not exist, that it has never existed, and that this concept has no counterpart as regards our everyday experience. In other words, the concept of nature as nature is a product of culture and occurs only as its opposite. Therefore, the concept of nature as nature assumes the 
existence of culture. It can be said that nature as nature is the product of a mind that is used to thinking in the categories of opposition.

So, nature as nature exists rather in our minds, in our imagination, and it is usually highly appreciated. The concepts that are the closest to it include wilderness, innocence, and purity-emphasizing the state of being immaculate, uncultivated, untouched by man. And although the word wilderness originated from the word "wild," which is associated with an uncomfortable sense of danger, in modern times it is its positive aspect that is recognized first and foremost; the untouched quality and innocence of nature as nature is emphasized. Undoubtedly, we need this concept since, having no counterpart in the actual world, it satisfies our vital need: the longing for something primal and intact. This need is so strong that the natural landscape is for us a part of nature as nature, which we long for to such an extent that-eagerly accepting the illusion-we ignore the traces of human activity that are present in the landscape.

As has been said, we humans don't have a firm and direct grasp on nature as nature. If we wanted to find it, we would be seeking it in vain in a landscape painting, where the presented landscapes almost always include elements of human activity. I would like to illustrate this with a few pictures, starting with the painting The Clouds by John Constable. This picture seems to represent nature as nature-even more so given that it concerns a part of the world that is hardly attainable for man. Obviously, we have to do with a painter's work, and, as we know, even in mimetic art the artists have an unlimited potential for creating the shapes of the reality they represent. In this case, however, art historians who study the series of Constable's drawings of clouds almost univocally emphasize that in this painting the artist consciously restricted his invention, and his intention was not to create a subjective image but rather to represent a view of clouds. The word "view" assumes here its fundamental meaning.

We can agree that in the image of the clouds themselves we do not find human presence; nevertheless, it can be found beyond the picture: that of the viewer (both the artist and the recipient) without whom those clouds would not be a view, and therefore they would not constitute a landscape either. The concept of a landscape includes the viewer. The landscape is not a self-contained entity; it exists with reference to that viewer. This is true for both painted landscapes and actual ones. It is important to emphasize that the viewer, constituting a condition of the landscape, is never within it; he is always without, at some distance. The landscape is given for the eye, and the eye belongs to our sense of distance. Thus, we view an actual landscape in a way similar to that in which we view a painted one.

The eye does not modify the landscape and yet the landscape exists only in so far as it is given to the eye. This is why even in the case of a cloudy sky, the presence of a viewer implies that we are not being presented with nature as nature. The human pervades the non-human; we are in the human world, in the anthroposphere 
in which there is no room for the opposition of culture and nature in the strong sense, and nature as nature proves to be merely a projection of an ideal paradise as the primordial state with an air of nostalgia.

If we agree that nature as nature exists only as a notion, the landscapes that are called natural are, in fact, artifactual. We commonly encounter situations in which human artifacts produced for various reasons fill the world. So, now we shall replace the opposition of human/non-human with another one: artificial/natural. The constant process of filling the world with artifacts and of introducing them into the sphere described as nature (that which has been given) assumes different dimensions: from a simple addition of products, as it were, placing them in the surroundings, to the forms of advanced reshaping of that which is natural. Here I have in mind actions like marking out and maintaining mountain paths and trails, constructing power lines and networks, cable-cars, and buildings, cutting down trees or their branches, regulating rivers, creating gardens and parks, and, finally, intentionally reshaping whole landscapes. In the opposition of artificial/natural, whatever is natural is still highly appreciated while the artificial, especially if it refers to the so-called ordinary artifacts produced to satisfy practical needs, is treated with much less fondness.

As regards landscape painting, there are numerous pictures in which artifactual elements and natural ones are intertwined and merged, constituting a complex whole. The relations between artifacts and nature in landscape space are either specifically balanced or one of the factors dominates. In Constable's A Mill at Gallingham in Dorset we are presented with a balance. Moreover, while Gustave Eiffel's viaduct is perceived as a distinct intervention in the natural landscape, the old water mill that now belongs to the past assumes a value closer to that which we ascribe to nature itself and is treated with sentiment. In the case of Eiffel's work, we sense a strong contrast between nature and technology; in Constable's painting, the water mill, powered by an element of nature, remains in harmony with it. On the other hand, in another painting of the English landscapist, Dedham Lock and Mill, human artifacts present in the picture are clearly dominant, pushing nature-water, grass, and animals-to the margin; it is only the two larger trees on the right hand side that attract the viewer's attention.

So far, I have tried to show artifactual landscapes devoid of human figures. It is not easy to find such pictures among landscapes-artifacts are usually accompanied by people.

Humans are included in the landscape just like artifacts. They form a part of itdoing their jobs or relaxing. We can easily say that for them the landscape does not exist; they do not notice it since they are preoccupied with their activities. A landscape, no matter whether is it painted or real, exists in the eyes of the viewer, for the observer who is beyond the landscape, being neither its participant nor its part. 
The landscape comprising elements of nature, artifacts, and people busy with various forms of activity is the landscape we encounter in our experiences. It should be noted that many objects produced by men assume meanings that go far beyond their practical function. They assume symbolic, religious, historical, aesthetic, or social dimensions, and the nature accompanying them, included in the new connotational field, is "nature as culture." The landscape becomes a cultural landscape in which the opposition of culture/nature finds itself groundless.

I introduced the ideas of the natural landscape, the artifactual landscape, and the cultural landscape emphasizing the different grades of human presence in each of them. I also tried to prove that the basis of our understanding of the landscape is constituted by conceptual oppositions like human/non-human, artifactual/natural, culture/nature. However, landscapes occur rather in the space "between" these oppositions.

The oak tree called Bartek is the largest and one of the oldest trees found in Poland. It is now $30 \mathrm{~m}$ tall; it has a trunk with a girth of $9.85 \mathrm{~m}$ as well as a crown that spreads about $40 \mathrm{~m}$. It is hard to say how old the tree is: scientists claim it is about 650 years old while tradition suggests it is 1200 years old. The hollow fragments of its trunk have been filled with concrete; its branches have received telescopic supports, and a lightning protection system has been installed. Despite the rot infested trunk, Bartek is still a living tree. It can be situated in the space "between" nature and culture.

In order to say goodbye to the 19th century and welcome the 2oth, some Christian countries initiated special initiatives. Among others, in 1901 in Poland, a huge cross$15 \mathrm{~m}$ tall and $5.5 \mathrm{~m}$ wide-was constructed on top of Mt. Giewont $(1875 \mathrm{~m})$. Seen from afar, it is not particularly large, especially as compared to the huge mountain massif. However, it dominates due to its religious, symbolic meaning. In the landscape, the artifactual element and nature seem to belong to two totally independent orders. And yet, they interact-the cross attracts thunderbolts which, during the past 110 years, have significantly weakened its structure.

\section{Landscape and senses}

I have already stated that the landscape is given for the eye. Dictionaries define landscape as an expanse of scenery that can be seen in a single view or an extensive area of land regarded as being visually distinct. This is why landscape painting has come into being; painting is an art for viewing, and that is why we look at real landscapes in the way we have learnt while dealing with paintings. And although the landscape is sensually rich, the contribution of the remaining senses in perception is significantly diminished by the dominance of vision. That is doubly so given that the distance involved in the concept of visual perception grows enormously in the case of a landscape, for it encompasses an extensive view. Obviously, 
in this situation the "contact" senses, which require closeness, cannot be fully engaged.

Activation of the contact senses requires participation. Yet, is it possible to participate in a landscape? We have seen people represented in the landscapes by John Constable, who undoubtedly receive a whole range of sense perceptions, particularly tactile ones, but, at the same time, their sight is limited to a very small area. They do not see the landscape. It seems that for the participant of a landscape, the landscape disappears-being replaced by the environment. Here we can indicate swimming as an example of man's utmost submergence into the environment. This example allows us to realize that the landscape of a river, a lake, or a sea shore is given only to the person who is looking at it from afar. If he decides to take a plunge, that is, to destroy the distance, we can no longer speak of a landscape; rather, we are then dealing with environmental relations based on closeness. It is as if our senses of distance and those of contact cannot cooperate in harmony at their full capacity: when the eye dominates, the sense of touch is inhibited, and when touch takes over, the range of sight is reduced to the touched area.

Is, therefore, the landscape totally a product of visual culture, and does it exist only when it is watched? It might seem that the doubts which have been raised here will disappear when we use an urban landscape as an example. Obviously, we can watch a city from a distant hill or the highest floor of a skyscraper. However, when we speak of the urban landscape, we have in mind not its view from afar, but rather the network of its characteristic interrelations inasmuch as they are different from the suburban or farmland networks. In the former case, we really watch the landscape, remaining beyond it. In the latter case, we are included in the network of urban interrelations. Should we, therefore, speak of a landscape or rather of a city environment? Both of these words-landscape and environment-have something in common: they signify people's surroundings. For this reason, fairly inconspicuously, they overlap and have started being used interchangeably as synonyms. This, however, means that they lose their specific meaning. In the case of a landscape the surroundings are watched from a distance, while in the case of the environment the surroundings include the watcher themselves - they are so close that interaction is unavoidable.

\section{Aesthetic character of the landscape}

The aesthetic element seems to be essential for the landscape-we admire its beauty. Observing a landscape like a painting is completely situated in modern aesthetics, based on the autonomy of the object and disinterestedness of perception as the conditions of experiencing beauty. When we pass from the concept of landscape to the concept of environment, from the viewer to the participant, the aesthetic quality-though still important-assumes another meaning, closer to its etymology 
connected with aisthesis. In both cases the aesthetic quality means experiencing pleasure, but the focus is shifted. In the sense connected with disinterested perception, the pleasure comes from the contemplative (distanced) savoring of the object, while in the latter case the pleasure is generated by the interaction engaging all of one's senses, which is inevitable when immersed in an environment. The metaphor of the eye fully reflects the former kind of aesthetic quality, but to reflect the latter one it would be better to use the metaphor of touch-the only reciprocal sense, to touch means to be touched.

Here we should introduce the concept of experience in the broad sense, which follows from pragmatic aesthetics based on the philosophy of John Dewey. He understood experience as fully sensual and somatic, as an interaction between an organism and its environment, and it was not by chance that he used the very notion "environment," which became a term, a concept in philosophy much later, thanks to ecology. In experience understood in this way, the aesthetic, as a quality of every experience, is not in opposition to the practical. We experience the environment in its mutually complementary aspects.

Arnold Berleant distinguished three models of experience: contemplative, characteristic for modern aesthetics, active (Dewey, Merleau-Ponty, Bollnow), engaging the subject, and participative, based on an interaction between the subject incarnate and the environment. ${ }^{1}$ Participative experience became the principal concept of the environmental aesthetics developed by Berleant, which was better adjusted for our time than the traditional aesthetics developed in the $18^{\text {th }}$ century.

For us, it is important that Berleant tries to expand participative experience to include experience of the landscape as well. He admits that in landscape painting "the observer is removed from the scene and contemplates it from a distance," and that "such paintings illustrate the usual definition of landscape as 'a picture representing a section of natural, inland scenery' that reflects the conception of landscape as 'an expanse of natural scenery seen by the eye in one view'" (Berleant 2005, 5). Still, he finds it possible to revise this conception of landscape through a re-interpretation of landscape painting executed in the spirit of participative experience. He gives several arguments indicating that numerous works of this genre of painting "draw the viewer into the space as an invitation to visit," "incorporate the perceiver into their space, compelling involvement," "serve as an invitation, leading the viewer to enter the pictorial space," "through the effective use of pictorial qualities a painting crates the total sensory field of experience" (Berleant 2005, 10-11).2

This is just a fragment of an admittedly fairly convincing argument. Nevertheless, I think that attributing a power of turning a recipient into a participant to the painting is possible only in the language of metaphor. An invitation to take part is not

\footnotetext{
1 In my opinion, Dewey's conception of experience fully characterizes Berleant's participative model, for the description of which Deweyan terms like interaction, energy field, organism, environment, etc., were applied.

2 This conception appeared in Berleant's early works, particularly in Art and Engagement (1991).
} 
participation. Pictorial means of expression may evoke the impression of somatic and multi-sensual participation in a viewer only through mediation, which we do not encounter in a participative experience of the environment. ${ }^{3}$ Berleant does not introduce a terminological difference between the landscape and the environment. ${ }^{4}$ I believe, however, that it is worth maintaining this difference.

I am aware that my position may seem outdated and, maybe, too straightforward as compared with the-two decades old-advanced and sophisticated reflection over the landscape. It is, as it were, a step backwards. For much has been done to change our attitudes towards the landscape as a view examined as if it were a painting-passively and disinterestedly, from a remote, static observation point. But if we endow the landscape with the features that have been worked out by ecology, eco-philosophy, and environmental aesthetics in reference to the environment-features like interactivity, reciprocity, somatic and multi- sensual involvement-what will remain as the difference between a landscape and an environment? And if we treat these two concepts as synonyms, what will happen to the whole semantic load of the landscape as developed within modern aesthetics? It would be neither easy nor useful to get rid of it. The two meanings of the landscape, the older one as a view perceived by sight and the newer one as an engaging interaction, cannot be united, harmonized, and synthesized. If it were possible, the synthesis could constitute the essential difference between the category of landscape and that of environment. However, that is not the case; the new meaning abolishes the old one, replacing it outright.

It is better to preserve the concept of landscape in its historical, semantic shape, and the new meaning ascribed to it should be left where it emerged, that is, in reference to the environment. We need both of these concepts in their clear semantic distinction. That being said, I would prefer to leave the issue open. Hence, I will conclude my considerations with a few questions.

\section{Twilight of the landscape?}

It seems that the categories of landscape and environment are neither synonymous nor even complementary. Will the potential of the landscape run out with

3 Martin Seel, writing on aesthetic perception with reference to the history of aesthetics from Alexander Baumgarten to Theodor W. Adorno, claims that aesthetic perception consists in synesthesia; it differs from other forms of perception through special connection of all the senses. There are no aesthetic experiences limited to only one sense. Seel admits, however, that when we, for example, see an object, the other senses accompany or penetrate the sight as the projects of imagination (Vorstellungen) (Seel, 2000).

4 Like many other representatives of environmental aesthetics, J. Douglas Porteous perceives the need to distinguish "urban" and "nonurban" areas-landscapes and townscapes. Claiming that the "environment is the stage on which human activity is set" (Porteous 1996, 192), he uses the concept of environment interchangeably with the two kinds of "scapes." However, in my opinion, man's activity and his relation to the landscape on the one hand and the environment on the other hand are fundamentally different. 
the twilight of visual culture? The issue is not the fact that people will cease to admire views, but the theoretical capacity of the category of landscape. At present aesthetics is executing a radical transformation and primary importance is being assumed by categories like interaction instead of contemplation, participation instead of observation, involvement instead of passivity, multi-sensory character emphasizing touch instead of visuality, immediateness (immersion) in place of distance. Will these new categories allow us to preserve the concept of landscape? Will they emphasize those properties of the landscape that have gone unnoticed so far? Will attributing features characteristic of environment to the landscape not turn it into a redundant category?

\section{References:}

Berleant, Arnold. 1991. Art and Engagement. Philadelphia: Temple University Press.

Berleant, Arnold. 2005. Aesthetics and Environment: Variations on a Theme. Burlington: Ashgate.

Porteous, J. Douglas. 1996. Environmental Aesthetics. Routledge: London.

Seel, Martin. 2000. Ästhetik des Ercheinens. Vienna: Carl Hanser Verlag München. 